\title{
Immunohistochemical and Zymographical Expressions of Matrix metalloproteinase-2 (MMP-2) and Matrix Metalloproteinase-9 (MMP-9) in Bovine Papillomatous Digital Dermatitis
}

\author{
๑ Ahmet AKKOC $^{1}$
}

1Bursa Uludag University Faculty of Veterinary Medicine, Department of Pathology, Gorukle, Bursa, Turkey.

\author{
Received 05.09.2019 Accepted 10.11.2019
}

\begin{abstract}
Bovine digital dermatitis (BDD) is an important health issue of dairy cattle. Painful papillomatous tissue proliferations are observed in hind feet of affected animals. The pathogenesis of this papillomatous lesion in BDD is still poorly understood. Matrix metalloproteinases are a group of enzymes for tissue remodeling in both health and disease. In the submitted study, immune expressions of MMP-2 and MMP-9 enzymes and their zymographic activities were evaluated. Skin samples were obtained from 10 healthy and 10 dairy Holstein frisean cattle from local slaughterhouse. Higher immune staining of MMP-2 and MMP-9 were found in BDD samples when compared with healthy skin samples. The differences observed in MMP-2 and MMP-9 staining intensity and distributions were statistically significant ( $\mathrm{p}<0.05, \mathrm{p}<0.05$ respectively). Zymographic analyses revealed skin samples from both healthy and affected animals had pro-active and active forms of MMP2 and MMP-9 enzymes. Immunohistochemistry results indicate that significant increase in MMP-2 and MMP-9 enzymes in BDD samples. These results suggest MMP-2 and MMP-9 might have roles in the pathogenesis of BDD.
\end{abstract}

Key words: Bovine digital dermatitis, Matrix metalloproteinases, immunohistochemistry, zymography

\section{Introduction}

Bovine Digital Dermatitis (BDD) is a common problem of dairy cattle. BDD results in large welfare and economic problems; hence prevention and treatment of the disease is of great importance. ${ }^{1}$ The main clinical feature of BDD is lameness resulting from a papillomatous lesion above the coronary band between the heel bulbs in hind feet. ${ }^{2}$ The etiology and pathogenesis have not yet been clarified. Even though the majority of evidence suggests involvement of spirochetes, Koch's postulates has not been fulfilled for the exact pathogenesis. Histologically, hyperproliferative (acanthotic) changes and inflammatory lesions are cardinal features of affected epidermis and dermis, respectively. ${ }^{3}$
Matrix metalloproteinases (MMPs) are a large family of zinc-containing endopeptidases, which are responsible for the tissue remodeling. ${ }^{4}$ MMP-2 and MMP-9 enzymes are prominent members of the MMP family. Keratinocytes, dermal fibroblasts, endothelial cells and other members of the skin synthesize various MMPs. ${ }^{5}$ In some circumstances, MMPs expression profile in the epidermis is elevated. ${ }^{6}$ The activities of MMPs manage many essential cellular events like proliferation, migration and adhesion. ${ }^{7,8}$ The roles of MMPs in hyperproliferative changes in skin is studied in humans. ${ }^{6}$ The upregulation of MMP-19 in proliferative phase not in neoplastic transformation phase of various skin disorders is demonstrated. ${ }^{7}$ Up to date, there are only three studies about MMPs and their contribution

\footnotetext{
* Corresponding author: Ahmet Akkoç, Bursa Uludag University Faculty of Veterinary Medicine, Department of Pathology,
} Gorukle, Bursa, Turkey. 
to bovine digital dermatitis .9-11 Elevated levels of MMP$12^{9}$ and MMP- $13^{10}$ enzymes in skin keratinocytes and dermal fibroblasts were demonstrated. Hypoxic regulation and increased amount of MMP-1, MMP-2 and MMP-13 enzymes were revealed in bovine dermal fibroblasts. ${ }^{11}$ The submitted study primarily aims to demonstrate immunohistochemical and zymographic expression of MMP-2 and MMP-9 enzymes in healthy and diseased skin samples of cattle suffering from papillomatous digital dermatitis. To the best of authors' knowledge, this study is the first documentation of the localization of MMP-2 and MMP-9 enzymes in healthy and diseased skin samples in cattle.

\section{Materials and Methods}

Ten control skin samples and ten papillomatous digital dermatitis samples were collected from local slaughterhouses. Classification of BDD lesions was performed according to a standardized scoring system. ${ }^{3}$ Skin samples were collected from hind feet lesions using an $8 \mathrm{~mm}$ biopsy punch. Half of the each biopsy samples were immersed in neutral buffered $10 \%$ formalin and processed routinely for histological examination. The other halves were kept in liquid nitrogen until the day of protein extraction. Sections of each sample were stained with Hematoxylin-Eosin.

\section{Immunohistochemistry}

Slides were stained immunohistochemically with the streptavidin biotin peroxidase method. Briefly, tissue sections ( $4 \mu \mathrm{m}$ in thickness) were mounted on poly-L-lysine coated slides and boiled in citrate buffer solution ( $\mathrm{pH}: 6)$ for antigen retrieval and endogenous peroxidase activity was inhibited in 3\% hydrogen peroxide solution for 5 minutes. Incubation with 1:100 diluted primary antibodies (MMP2: Ab37150, MMP-9: Ab38898, Abcam, Cambridge, MA) was performed for 1 hour at room temperature, and the slides were then incubated with the linking antibody and with the peroxidase conjugated streptavidin for 20 minutes at room temperature. Peroxidase activity was developed in diaminobenzidine (DAB) chromogen for $15 \mathrm{~min}$. Slides were counterstained with haematoxylin, dehydrated, and mounted. Bovine placenta was used as positive control for both enzymes. Negative control sections were incubated with PBS instead of the primary antibodies. Distribution and intensity of immunohistochemical stainings were used as key findings during scoring; In brief, the scores and their expression were 0 : unstained, + : faint, ++ : moderate, ++ + : strong, or ++++ : very strong.

\section{Protein Extraction}

Tissue samples (40 mg, wet weight) were homogenized in $1.5 \mathrm{~mL}$ extraction buffer (containing $10 \mathrm{mM}$ Tris $\mathrm{pH}$ 7.4, $150 \mathrm{mM} \mathrm{NaCl}, 1 \%$ Triton X-100) per gram of tissue using ice cold mortar and pestle. The homogenates were transferred to $1.5 \mathrm{~mL}$ centrifuge tubes and centrifuged at $13,000 \mathrm{xg}$ for 10 minutes at $4^{\circ} \mathrm{C}$, and the supernatant was collected, aliquoted into small volumes and stored at $-20^{\circ} \mathrm{C}$ until analyzed. Total proteins of each sample were calculated using commercially available protein determination kit (BCA-1, Sigma). Manufacturer's protocol was followed.

\section{Gelatin Zymography}

The activities of MMP-2 and MMP-9 enzymes were evaluated by gelatin zymography as described previously ${ }^{12}$ with $10 \%$ polyacrylamide gel containing $0.1 \%$ gelatine (G9136, Sigma) as substrate. Supernates were diluted 1:1 in zymogram sample loading buffer (161-0764, Bio-Rad, Hercules, CA, USA) and electrophoresed in non-reducing electrophoresis conditions at 150 volts for an hour using Mini-Protein ${ }^{\circledR}$ II electrophoresis unit (BioRad, USA). Then, gels were washed with Triton X-100 in zymogram developing buffer $\left(0.05 \mathrm{M}\right.$ Tris- $\mathrm{HCl} \mathrm{pH}: 8$ and $\left.5 \mathrm{mM} \mathrm{CaCl}_{2}\right)$ for at least 4 hours to remove sodium dodecyl sulphate (SDS) in the gel. Following washing step, gels were incubated with zymogram activating buffer $(0.05 \mathrm{M}$ Tris- $\mathrm{HCl} \mathrm{pH}: 8$ and $5 \mathrm{mM} \mathrm{CaCl}$ ) overnight at $37{ }^{\circ} \mathrm{C}$ and then stained with 0.1\% Coomassie blue R-250 in methanol:acetic acid:distilled water (v:v:v-4.5:1:4.5) for 4 hours on a rotary shaker at room temperature. The gels were destained until the bands were clearly visible and digital images were acquired using digital gel imaging system (DNR ChemiBis, Israel) and evaluated by GelQuant Pro program.

\section{Statistical Analysis}

Statistical significance of results was determined by using Student $t$ test when BDD groups were compared with their respective controls. The value of $\mathrm{p}<0.05$ was considered to be significant.

\section{Results}

\section{Gross lesions}

Any lesion was observed in control skin samples. Hairy papillomatous skin proliferations (Figure-1) were evident in cattle suffering from digital dermatitis.

\section{Histopathology}

The epidermis with keratinized squamous epithelial cells and uniform dermal structures were observed in control slides from healthy animals (Figure-2). Severe acanthosis, hyperkeratosis and infiltrations of polymorphonuclear and mononuclear leucocytes were the major histopathological findings in diseased skin samples (Figure-3).

\section{Immunohistochemistry}

Healthy skin samples showed MMP-2 immune reactivity in epidermis except the basal epithelial layer. The staining was in granular appearance and located in the cytoplasm of epithelial cells (Figure-4). The endothelial cells of blood vessels and stromal fibroblasts in dermis were stained posi- 
tively with the MMP-2 antibody. MMP-2 enzyme was also present in the epithelium in BDD to a greater extent than normal epidermis in controls. In the control skin samples MMP-9 immune expression was present in epithelial cells and dermal fibroblasts. The endothelial cells were not stained with anti-MMP-9 antibody. The epithelial cells in epidermis, inflammatory cells, vascular endothelial cells and stromal fibroblasts displayed positive reaction against MMP-9 antibody in BDD samples. MMP-2 and MMP-9 enzymes stained more intensely in BDD samples when compared with healthy tissues. Results were summarized in Table-1. Staining specificity was checked by using positive control tissue (bovine placenta). Trophoblastic cells and endothelial cells were strongly positive against to MMP-2 and -9.

Table-1 The average staining scores of MMP-2 and MM-9 healthy and diseased skin samples. $(\mathrm{P}<0.05)$. All data are shown as mean $\pm \mathrm{SD}$.

\begin{tabular}{|c|c|c|}
\hline & Healthy samples & Diseased samples \\
\hline MMP-2 scores & $+1.4( \pm 0.2)$ & $+2.5( \pm 0.5)$ \\
\hline MMP-9 scores & $+1.1( \pm 0.3)$ & $+2.2( \pm 0.3)$ \\
\hline p value & $<.05$ & $<.05$ \\
\hline
\end{tabular}

\section{Gelatin Zymography}

Gelatin zymograms revealed existence of both active and pro-active forms of MMP-2 and MMP-9 enzymes in bovine skin samples. Gelatinolytic bands corresponding to MMP-2 and MMP-9 enzymes were observed as clear lines.

\section{Discussion}

The exact pathogenesis of bovine digital dermatitis is still unknown. Further and detailed studies are needed to completely understand the mechanism. This study aims to scrutinize possible roles of MMP-2 and -9 enzymes in that pathogenesis by immunohistochemistry and zymography. In the first step, bovine papillomatous digital dermatitis was confirmed by histopathological evaluation and then immunohistochemistry was used to examine enzyme localizations. Light microscopic findings were in accordance with previous study. ${ }^{3}$ Immunohistochemically, keratinocytes, dermal fibroblasts and endothelial cells of blood vessels were positively stained and elevated intensity of staining of MMP-2 and MMP-9 enzymes were found in papillomatous dermatitis samples when compared with healthy equivalents. The statistically significant differences $(\mathrm{p}<, 05)$ (in both MMP-2 and MMP-9) were found between healthy and diseased samples.

The role of various cells in the pathogenesis of BDD in the context of MMPs has been reported by some researchers. ${ }^{9-11}$ Previous studies used keratinocyte and dermal fibroblast cell culture models and reported upregulated MMP-12, MMP-1, -2 and -13 levels in in response to some inflammatory molecules. It is clear from their studies; increased MMP enzymes may play important roles in the pathogenesis of bovine digital dermatitis. In a previous study, 13 challenge of dermal fibroblasts with LPS caused increased secretion of MMP-2 and MMP-9 enzymes into culture medium. Parallel to these findings, in the submitted study, it was observed that immunostaining intensity of MMP-2 and - 9 enzymes in diseased skin samples were increased. The higher amounts of these enzymes are likely to be effective in proliferation of cells in the tissue. In a study, ${ }^{7}$ it was shown that expression of MMP-19 in epidermal lesions changes time dependently. Unfortunately, in the submitted study, the sampling was done once and the enzyme profiles before or after sampling was not known. Zymography is an extremely useful method for evaluating the activity of MMPs. In the submitted study, clear proteolytic bands equals to $72 \mathrm{kDa}$ MMP-2and 92kDa MMP-9 enzymes were captured in gelatin zymograms. Thus, some of the enzymes observed in immunostaining were found to be in active form. MMP-2 and MMP-9 enzymes were stimulated in vitro during zymographic analysis; therefore, the clear bands obtained in zymograms matched the active forms of MMP-2 and MMP-9. It can be conclude that active forms of both enzymes can cleave cellular connections and facilitate epithelial cell migrations for hyperplastic lesions in situ.

As the numbers of MMP studies are very few, the data submitted herein can bring new approaches to understand the disease process and successful treatment options since MMPs can be easily inhibited by certain chemicals.

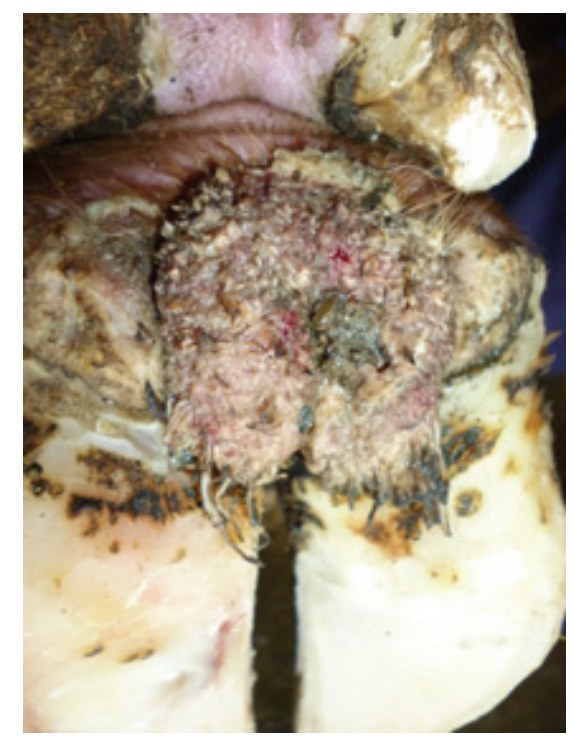

Figure-1: Papillomatous tissue proliferation in the hind foot of cattle. 


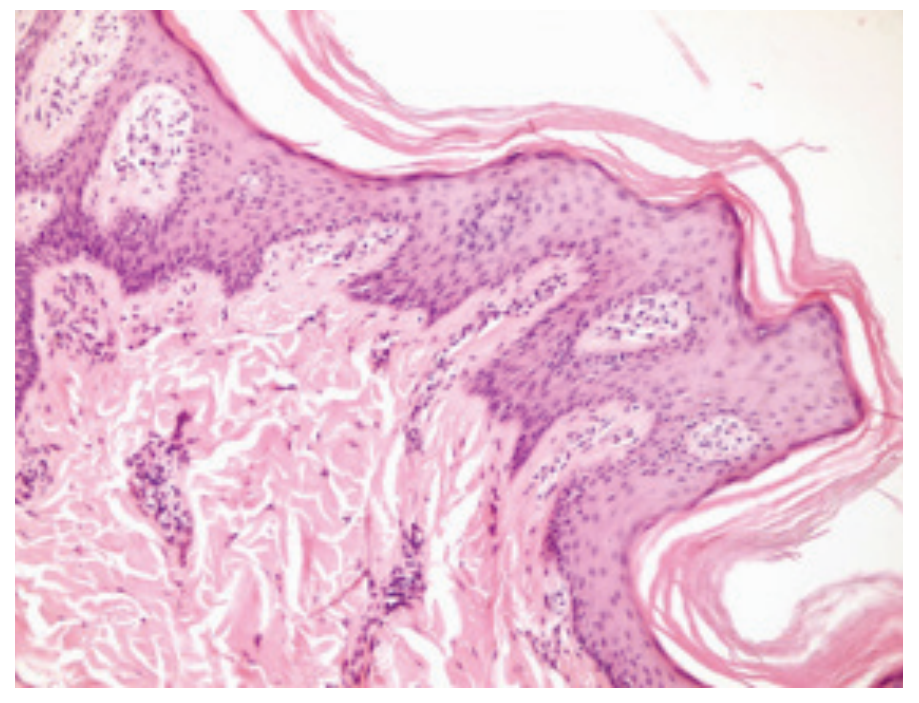

Figure-2: Histopathological appearance of healthy skin, please note that clear separation of keratin layer, epidermis and dermis. Hematoxylin-eosin staining, 100X magnification.

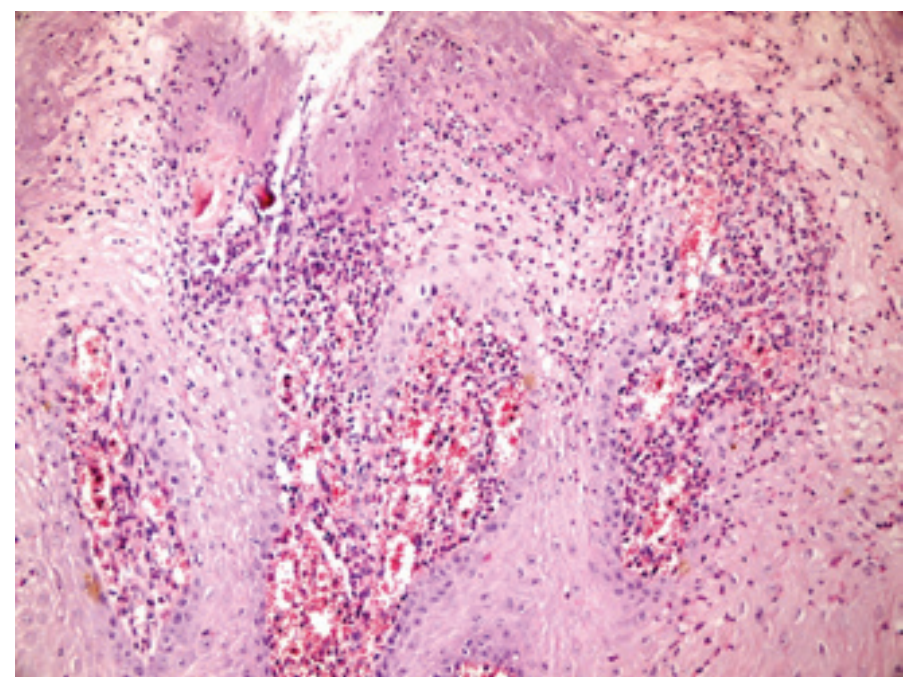

Figure-3: Severe inflammatory changes in dermis and proliferative reaction in epidermis. Hematoxylin-eosin staining. 100X magnification.

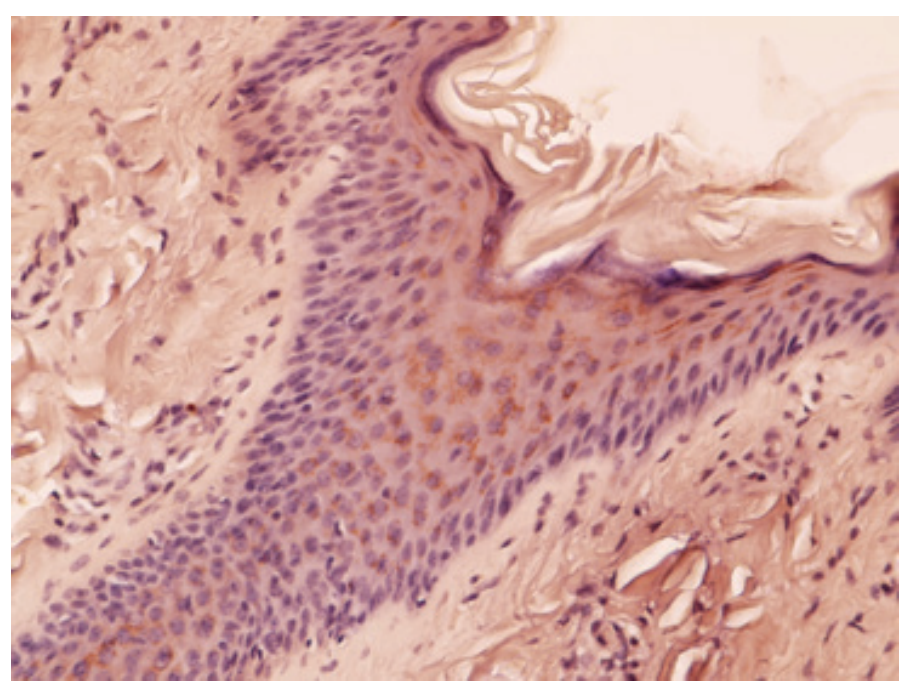

Figure-4: MMP-2 immunostaining in healthy skin, streptavidin biotin peroxidase method. DAB chromogen. 200X magnification. Please note that mild cytoplasmic staining intensity $(+2$ score).

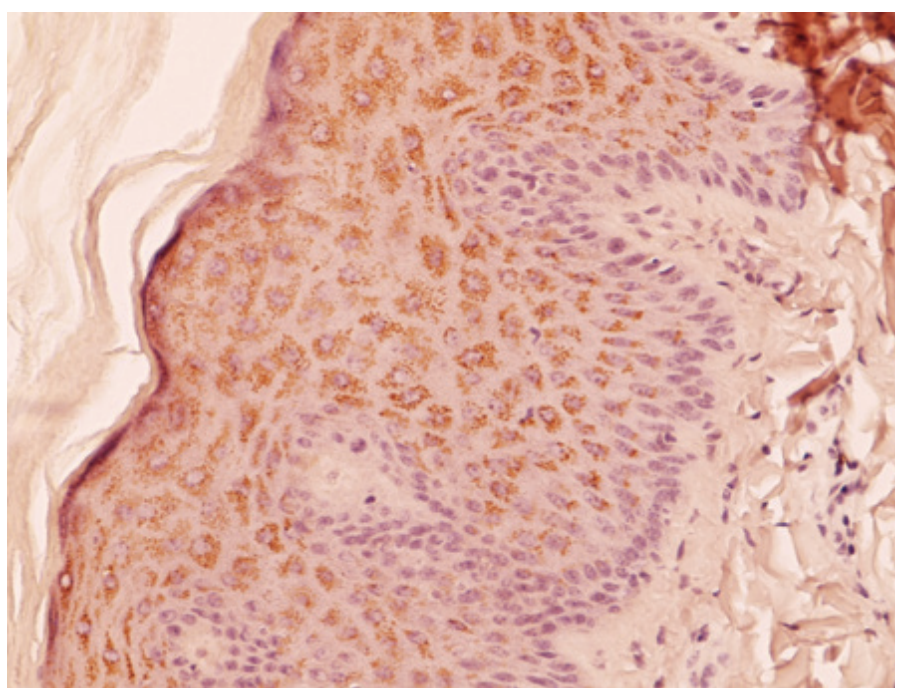

Figure-5: Strong MMP-2 immunostaining in BDD sample, streptavidin biotin peroxidase method. DAB chromogen. 400X magnification. Please note that the cytoplasmic staining intensity (+4 score).

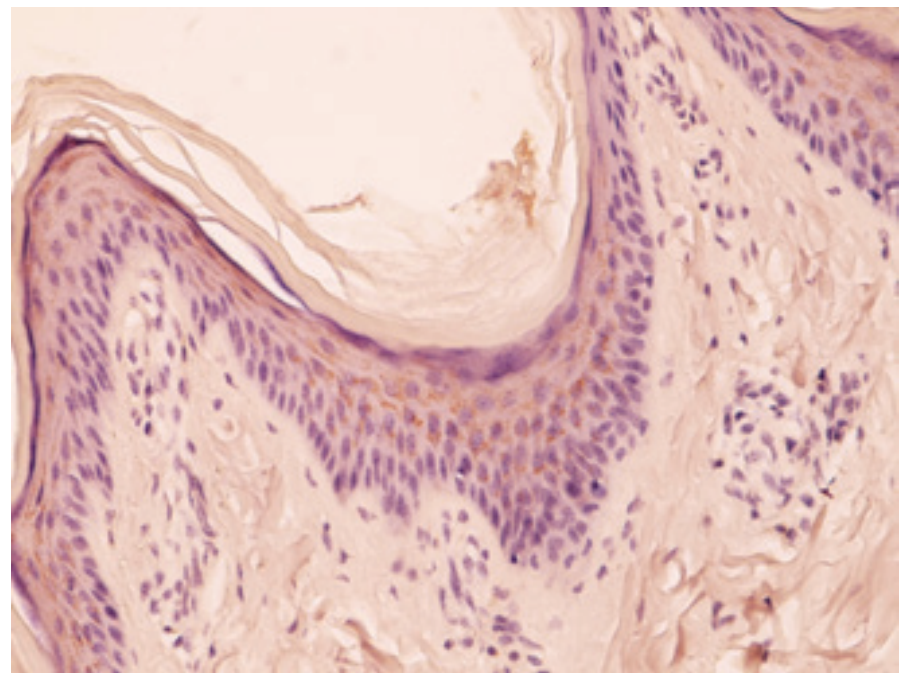

Figure-6: MMP-9 immunostaining in healthy skin, streptavidin biotin peroxidase method. DAB chromogen. 200X magnification. Please note that mild cytoplasmic staining intensity $(+2$ score).

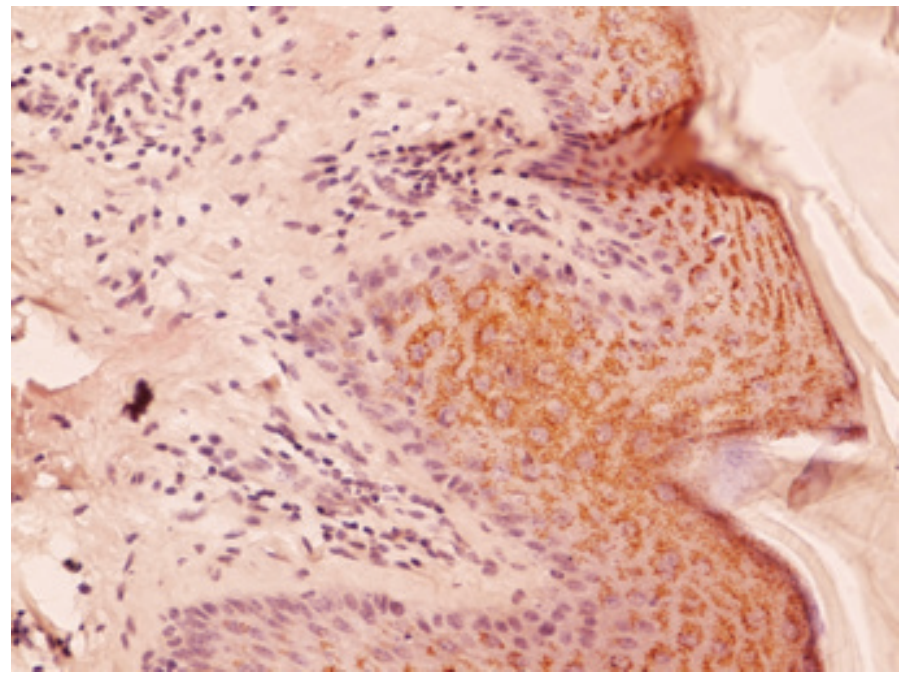

Figure-7: Strong MMP-9 immunostaining in BDD sample, streptavidin biotin peroxidase method. DAB chromogen. 200X magnification. Please note that the cytoplasmic staining intensity (+4 score). 


\section{References}

1- Berry SL. Diseases of the digital soft tissues. Vet Clin North Am Food Anim Pract. 2001; 17:129-142.

2- Blowey RW, Sharp MW. Digital dermatitis in dairy cattle. Vet Rec. 1988; 21;122(21):505-508.

3- Döpfer D, Koopmans A, Meijer FA et al. Histological and bacteriological evaluation of digital dermatitis in cattle, with special reference to spirochaetes and Campylobacter faecalis. Vet Rec. 1997; 140:620-623.

4- Nagase H, Woessner JF. Matrix metalloproteinases. J Biol Chem. 1999; 274:21491-21494.

5- D’Armiento J, DiColandrea T, Dalal SS et al. Collagenase expression in transgenic mouse skin causes hyperkeratosis and acanthosis and increases susceptibility to tumorigenesis. Mol Cell Biol. 1995;15(10):5732-5739.

6- Akgül B, Pfefferle R, Marcuzzi GP et al. Expression of matrix metalloproteinase (MMP)-2, MMP-9, MMP13, and MT1-MMP in skin tumors of human papillomavirus type 8 transgenic mice. Exp Dermatol. 2006;15(1):35-42.

7- Impola U, Toriseva M, Suomela S et al. Matrix metalloproteinase-19 is expressed by proliferating epithelium but disappears with neoplastic dedifferentiation. Int J Cancer. 2003;103(6):709-716.

8- Page-McCaw A, Ewald AJ, Werb Z. Matrix metalloproteinases and the regulation of tissue remodelling. Nat Rev Mol Cell Biol. 2007;8(3):221-33.

9- Evans NJ, Brown JM, Scholey R et al. Differential inflammatory responses of bovine foot skin fibroblasts and keratinocytes to digital dermatitis treponemes. Vet Immunol Immunopathol. 2014;161(1-2):12-20.

10- Scholey RA, Evans NJ, Blowey RW et al. Identifying host pathogenic pathways in bovine digital dermatitis. Vet. J. 2013; 197, 699-706.

11- 11- Van Diest Pj, van Dam P, Henzen-Logmans SC et al. A scoring system for immunohistochemical staining consensus report of the task force for basic research of the EORTC-GCCG. European Organization for Research and Treatment of Cance Gynaecological Cancer Cooperative Group. J Clin Pathol. 1997; 50
(10):801-804.

12- Hirayama K, Yokota H, Onai R et al. Detection of matrix metalloproteinases in canine mammary tumours: analysis by immunohistochemistry and zymography. J. Comp. Pathol. 2002;127: 249-256.

13- Akkoc A, Kahraman MM, Vatansever A et al. Lipopolysaccharide (LPS) induces matrix metalloproteinase-2 and -9 (MMP-2 and MMP-9) in bovine dermal fibroblasts. Pak Vet J. 2016; 36:189-193. 